\title{
Clasificación del riesgo de lavado de activos y financiación del terrorismo en Colombia en 2019*
}

\author{
Classification of the risks of money laundering and terrorist financing in Colombia in 2019
}

Classificação do risco de lavagem de dinheiro e financiamento do terrorismo na Colômbia em 2019

\author{
Henry Sebastián Rangel Quiñonez a ${ }^{\mathrm{a}}$ \\ Universidad Santo Tomás, Colombia \\ henry.rangel@ustabuca.edu.co \\ ORCID: https://orcid.org/0000-0002-6745-6753 \\ Gilma Barrera Gómez \\ Financiera Comultrasan, Colombia \\ ORCID: https://orcid.org/0000-0001-6143-3625 \\ Oscar Mauricio Gómez Sánchez \\ Financiera Comultrasan, Colombia \\ ORCID: https://orcid.org/0000-0002-3333-9671
}

DOI: https://doi.org/10.11144/Javeriana.cc22.crla

Recibido: 13/04/2021

Aceptado: 14/09/2021

Publicado: 31/12/2021

\section{Resumen:}

El lavado de activos y el financiamiento del terrorismo son actividades ilícitas que perjudican el crecimiento y la estabilidad económica de un país; estos delitos están relacionados con el aumento de la pobreza y la violencia por lo que su control debe ser riguroso. Esta investigación diseña un modelo de segmentación basado en el factor jurisdicción que permite identificar las ciudades de mayor exposición al riesgo del Lavado de Activos y Financiación del Terrorismo en Colombia. Para dar cumplimiento a lo anterior, se recolectó la información disponible para los 1.122 municipios del país, proveniente de fuentes externas como la Fiscalía General de la Nación, el Observatorio de Drogas de Colombia e Indepaz. Los resultados reflejan que el 85\% de los municipios son clasificados como riesgo bajo, $15 \%$ como riesgo medio y menos del 1\% como riesgo alto en lavado de activos.

Códigos JEL: C38, C43, K49.

Palabras clave: Riesgos LAFT, estadística multivariada, análisis de conglomerados, factor jurisdicción, economía subterránea.

\section{Abstract:}

Money laundering and terrorist financing are illicit activities that harm the growth and economic stability of a country; these crimes are related to the increase in poverty and unemployment and their control must be rigorous. This research designs a segmentation model based on the jurisdiction factor that allows identifying cities with the greatest exposure to the risk of money laundering and financing of terrorism in Colombia. To comply with the above, the information available for the country's 1122 municipalities was collected from external sources such as the Attorney General's Office, the Colombian Drug Observatory, and Indepaz. The results show that $85 \%$ of the municipalities are classified as low risk, $15 \%$ as medium risk and less than $1 \%$ as high risk in money laundering. EL Codes: C38, C43, K49.

Keywords: ML/TF risks, multivariate statistics, cluster analysis, jurisdiction factor, underground economy.

\section{Resumo:}

O branqueamento de capitais e o financiamento do terrorismo são actividades ilícitas que prejudicam o crescimento e a estabilidade económica de um país; estes crimes estão relacionados com o aumento da pobreza e do desemprego e o seu controlo deve ser rigoroso. Esta investigação desenha um modelo de segmentação baseado no factor de jurisdição que permite identificar as cidades com maior exposição ao risco de branqueamento de capitais e financiamento do terrorismo em Colômbia. Para cumprir o acima exposto, foram coletadas as informações disponíveis para os 1.122 municípios do país de fontes externas, tais como a ProcuradoriaGeral da Nação, o Observatório Colombiano da Droga e Indepaz. Os resultados mostram que 85\% dos municípios são classificados como de baixo risco, $15 \%$ como de médio risco e menos de $1 \%$ como de alto risco em matéria de branqueamento de capitais.

Códigos JEL: C38, C43, K49.

Notas de autor

\footnotetext{
a Autor de correspondencia. E-mail: henry.rangel@ustabuca.edu.co
} 
Palavras-chave: Riscos de BC/FT, estatística multivariável, análise de agrupamento de dados, fator de jurisdição, economia subterránea.

\section{Introducción}

El lavado de activos es un delito que busca dar apariencia de legitimidad a recursos de procedencia ilícita; ha sido una de las estrategias más comunes para ocultar el verdadero origen del dinero y de los activos de una persona natural o jurídica (Albanese, 2012; Levi \& Reuter, 2006). Los recursos obtenidos a través de actividades ilegales buscan, por medio de los diferentes sectores de la economía, permear el flujo económico de la nación y afectan directamente al sector financiero y cooperativo (Bayona-Rodríguez, 2019). Por su parte, la financiación del terrorismo se define como el aporte económico que proporciona un individuo o una organización con recursos de fuentes legales o ilegales, destinados a apoyar o financiar una estructura criminal o delincuencial (Romaniuk, 2014).

Tanto el lavado de activos como la financiación del terrorismo han logrado desestabilizar economías nacionales y su mayor impacto recae en las llamadas economías pequeñas (Rocha-Salazar, Segovia-Vargas, \& Camacho-Miñano, 2021). En Colombia estos delitos han causado efectos nocivos como el aumento de la pobreza, la violencia, el desempleo y la corrupción. Estas problemáticas surgen porque el Estado colombiano no logra controlar estas conductas punibles y requiere adoptar algunas medidas de seguridad inmediatas para frenar estos delitos; los cuales son castigados en el Código Penal con sanciones económicas y privativas de la libertad (Sintura, 2010). Pese a la normativa vigente, existe una necesidad inminente para que los mecanismos de control identifiquen la procedencia de los delitos fuentes del Lavado de Activos y Financiación del Terrorismo (LAFT).

Entidades de control como la Superintendencia Financiera, la Unidad de Información y Análisis Financiero (UIAF), la Superintendencia de Sociedades, entre otras, dan libertad a las entidades financieras para que adopten e implementen métodos para identificar y prevenir el LAFT. Esto evidencia la importancia de identificar el riesgo de la existencia del LAFT en los municipios de Colombia para así diseñar acciones de prevención a la medida en cada ciudad. Sin embargo, los estudios internos de las entidades financieras pequeñas suelen ser poco técnicos, por ende, ineficientes para detectar el ingreso de dineros producto de actividades ilícitas o con destino a financiar el terrorismo, y les acarrea enormes sanciones económicas por parte de los organismos de control. Lo anterior justifica la necesidad de inspeccionar nuevas estrategias científicas estandarizadas que permitan medir el riesgo de lavado de activos por ciudad, de tal forma que las entidades financieras puedan implementar medidas rigurosas en los municipios más riesgosos, optimizando los recursos invertidos en la prevención del LAFT y evitando sanciones (Puyana, 1999).

Esta investigación implementa una metodología para el cálculo del riesgo LAFT en ciudades de Colombia a partir de revisión de literatura y a criterio experto de funcionarios de una cooperativa colombiana con más de 70 años en el mercado financiero. Los datos utilizados son de libre acceso, así como el software estadístico implementado. Esta metodología junto con la aplicación de una técnica estadística ampliamente documentada y estandarizada puede ser replicada por parte de otras instituciones financieras en distintas regiones y otros periodos de tiempo.

\section{Historia del lavado de activos y financiación del terrorismo}

El lavado de activos es considerado un delito trasnacional que ha tenido un fuerte impacto en Colombia desde la década de 1970. Sus orígenes datan de la Edad Media cuando la usura fue declarada un delito y los banqueros y mercaderes empezaron a ocultar el origen de los ingresos de estas operaciones. Aunque, no fue hasta 1920 en el que este delito tomó el nombre de "lavado" pues el famoso mafioso estadounidense, al Capone, vio la 
oportunidad de disfrazar la fuente de sus recursos provenientes de manera ilícitas mediante la creación de una empresa dedicada a la lavandería de ropa (Ulloa, 2018; Levi, 2020).

En la década de 1970 aparece el narcotráfico como uno de los principales delitos para el lavado de dinero con recursos que entraban al torrente financiero sin ningún control. En Colombia, tuvo su apogeo en la década de 1980 con el surgimiento del cartel de Medellín, grupo que ingresaba dólares producto de la venta de estupefacientes en Estados Unidos (Rocha, 2001, 2014). Después se crearon otras organizaciones como el Cartel de Cali, Cartel de la Costa Caribe, Cartel de Bogotá, Cartel del Norte del Valle y algunas bandas criminales como los Rastrojos, Urabeños, Águilas Negras, los Machos, Cordillera y Alta Guajira (Bagley, 2000; Díaz \& Sánchez, 2004); los recursos de estas organizaciones se reflejaron en aumento de los indicadores de la economía y provocaron distorsiones macroeconómicas como inflación y problemas sociales de sicariato y violencia. El lavado de activos en Colombia se ha reducido desde la firma del Acuerdo de paz entre el Gobierno y el grupo insurgente FARC en 2016. Este Acuerdo tenía como fin evitar más víctimas y violencia en el país (Gobierno Nacional, 2016) e incluía la sustitución de cultivos ilícitos y la entrega de dinero producto del narcotráfico. Aunque al presentarse el desarme de esta organización criminal se crearon otras estructuras que persisten con este tipo de delitos.

A partir de 2013, Colombia ha venido implementando la Evaluación Nacional del Riesgo de LAFT, sus resultados han permitido conocer y entender cómo está el país en materia de riesgo de LAFT y así implementar un plan de acción que fortalezca los controles y las estrategias para mitigar el riesgo de estos delitos, con el fin de proteger la economía, la imagen del país y el bienestar de los colombianos. La última Evaluación Nacional del Riesgo (ENR) de 2019 fue liderada por la Unidad de Información y Análisis Financiero (UIAF), en esta evaluación se realizó una calificación del Nivel de Riesgo existente en Colombia frente al lavado de activos y la financiación del terrorismo (Rodríguez \& Castro, 2020). La metodología de evaluación utilizada es la del Banco Mundial, que consiste en calificaciones objetivas basadas en juicio de expertos, quienes sustentan sus calificaciones en información cuantitativa y cualitativa, estas evaluaciones son realizadas por personas con gran experiencia en ALA/CFT -Sistema Nacional Anti Lavado de Activos y Contra la Financiación del Terrorismo- (MinJusticia, 2015).

La evaluación nacional del riesgo (ENR) fue implementada en cuatro fases: 1) recopilación de información suministrada por las entidades vigiladas, 2) realización del primer taller para evaluar las amenazas y vulnerabilidades en los sectores sujetos de estudio, 3) presentación de los resultados obtenidos del taller en el que se plantearon políticas públicas, y 4) creación y puesta en marcha de las nuevas políticas. Para el caso del lavado de activos (LA), la calificación final en Colombia fue "medio-alto", el cual se distribuye en las amenazas con calificación "medio-alto" y para las vulnerabilidades existentes en el LA la calificación fue "medio". La calificación de las amenazas se determinó según los delitos cometidos relacionados con el LA; las vulnerabilidades son determinadas por las falencias encontradas en la calidad y efectividad de los controles establecidos, entre ellas se evidencia la falta de personal capacitado en temas de LA y la falta de infraestructura tecnológica (UIAF, 2019).

Para el caso de la Financiación del Terrorismo (FT) la calificación general para Colombia fue "medio", las amenazas se calificaron en "medio-baja" y las vulnerabilidades se calificaron como "medio-altas". Las amenazas se derivan de los recursos provenientes de la minería ilegal, tráfico de estupefacientes y el testaferrato; actividades criminales con un alto impacto en Colombia. En el caso de las vulnerabilidades, estas se identificaron teniendo en cuenta la baja efectividad de los reportes de operaciones sospechosas relacionadas con FT, los bajos recursos asignados para combatir el FT y algunos factores geográficos y demográficos que favorecen las condiciones para que se mantenga el FT (UIAF, 2019).

Por otra parte, la empresa de consultoría Lozano Consultores ha realizado investigaciones privadas que dan cuenta del riesgo a nivel departamental de la existencia de lavado de activos (Lozano, 2008). Esta entidad realizó el estudio con noticias publicadas en medios de comunicación desde el año 2010; los resultados concluyen que Cundinamarca, Antioquia, Valle del Cauca, Atlántico y el Huila son los departamentos 
con mayor riesgo. Si bien existen estudios para Colombia sobre el LAFT, corresponden a nivel país y departamento, dejando un vacío a nivel municipal que pretende ser llenado en esta investigación.

\section{Metodología}

Esta investigación sigue un enfoque descriptivo basado en una técnica multivariada aplicada a datos públicos de la Fiscalía General de la Nación (Fiscalía, 2020), Indepaz (2021) y el Observatorio de Drogas de Colombia (2021). Para empalmar y unificar las bases de datos de estas instituciones se utilizó el código DANE de los municipios (variable V1). Ver tabla 1. Las variables V1, V2 y V3 sólo se tuvieron en cuenta para el preprocesamiento; no en la implementación de la agrupación. El análisis de los conglomerados permitió agrupar las ciudades que presentan homogeneidad según el nivel de procesos o delitos asociados al lavado de activos. Para esta clasificación se utilizaron las variables relacionadas en la tabla 1 propuestas a criterio experto luego de entrevistas a dos funcionarios de una entidad financiera con más de 70 años de experiencia en el sector, además se consultó el artículo 323 del Código Penal colombiano (Ley 599 de 2000) que define los delitos asociados al LAFT.

TABLA 1

Variables contempladas en el estudio

\begin{tabular}{|l|c|}
\hline \multicolumn{1}{|c|}{ Variable } & Código \\
\hline Código DANE del municipio & V1 \\
\hline Nombre completo del municipio & V2 \\
\hline Iniciales del municipio & V3 \\
\hline Número de grupos armados, 2018 & V4 \\
\hline Hectáreas de cultivos ilícitos, 2019 & V5 \\
\hline Número de procesos por minería ilegal, 2019 & V6 \\
\hline Número de procesos contrabando, 2019 & V7 \\
\hline Número de procesos por contrabando de hidrocarburos y derivados, 2019 & V8 \\
\hline Número de procesos contra la administración pública, 2019 & V9 \\
\hline Número de procesos por delitos contra el sistema financiero, 2019 & V10 \\
\hline Número de procesos por enriquecimiento ilícito, 2019 & V11 \\
\hline Número de procesos por extorsión, 2019 & V12 \\
\hline $\begin{array}{l}\text { Número de procesos por favorecer el contrabando de hidrocarburos y } \\
\text { derivados, 2019 }\end{array}$ & V13 \\
\hline Número de procesos por favorecer y facilitar el contrabando, 2019 & V14 \\
\hline $\begin{array}{l}\text { Número de procesos por financiación del terrorismo y administración de } \\
\text { recursos relacionados con actividades terroristas, 2019 }\end{array}$ & V15 \\
\hline Número de procesos por fraude aduanero, 2019 & V16 \\
\hline Número de procesos por rebelión, 2019 & V17 \\
\hline Número de procesos por secuestro y extorsivo, 2019 & V18 \\
\hline Número de procesos por tráfico de armas, 2019 & V19 \\
\hline Número de procesos por tráfico de drogas, 2019 & V20 \\
\hline Número de procesos por tráfico de migrantes, 2019 & V21 \\
\hline Número de procesos por tráfico de niños y adolescentes, 2019 & V22 \\
\hline Número de procesos por trata de persona, 2019 & V23 \\
\hline Número de procesos por concierto para delinquir, 2019 & V24 \\
\hline
\end{tabular}

Fuente: elaboración propia.

Como parte del preprocesamiento de datos se optó por clasificar previamente a 30 ciudades como municipios de alto riesgo debido a que estos presentaban reportes de más de 550 delitos fuente de LAFT. En pruebas previas se determinó que de no excluir estas ciudades no había una correcta división de los municipios, pues el algoritmo discriminaba solamente a estas 30 ciudades del resto. El proceso de clasificación usado fue un algoritmo no jerárquico $\mathrm{k}$-medias a través del lenguaje de programación $\mathrm{R}$ (ver tabla $\mathrm{A} 1$ ). Así se logró la distribución geoespacial de la clasificación de riesgo LAFT en tres conglomerados (alto, mediano y bajo riesgo) y se realizó la representación de la distribución geográfica de los diferentes municipios de Colombia a través de un mapa diseñado en Arcmap.

Métodos no jerárquicos. Según Peña (2002), para la aplicación del método de clasificación no jerárquico se debe conocer previamente el número de conglomerados a generar, el algoritmo de clasificación no jerárquico 
más conocido es el K-Medias. Por otra parte, Díaz (2012) señala los pasos a aplicar en este tipo de método son: 1) calcular los centroides de forma aleatoria de cada grupo, 2) tomar cada individuo y asignar al centroide más cercano, 3) calcular el nuevo centroide, según los datos agrupados, y 4) repetir los pasos 2 y 3 hasta que los casos (municipios) dejen de moverse de un grupo a otro.

Método de K-Medias. Díaz (2012) define el procedimiento de agrupamiento de K medias como aquella técnica que permite fraccionar un conjunto de elementos en un número de grupos de acuerdo con los siguientes criterios: 1) seleccionar los centroides de los grupos que pueda reducir la distancia de cada elemento a ellos, y 2) asignar cada elemento al grupo verificando que el Centroide este más cercano al grupo. Dicho autor establece la agrupación por el método k-medias de la ecuación 1.

$$
D_{(i, l)}=\left(\sum_{j=1}^{p}\left(X_{i, j}-\bar{X}_{(l) j}\right)^{2}\right)^{1 / 2}
$$

Por otro lado, el componente de error de la partición está dado por la ecuación 2

$$
\mathrm{E}\{P(n, K)\}=\sum_{i=1}^{n}[D(i, l(i))]^{2}
$$

donde $l(i)$ es el grupo que contiene al $i$-ésimo individuo, y $D(i, l(i))$ es la distancia euclidiana entre el individuo $i$ y el centroide del grupo que contiene al individuo y $X_{i j}$ es el valor del $i$-ésimo elemento sobre la $j$-ésima variable; con $i=1, \ldots, n$ y $j=1, \ldots, p$. La media de la $j$-ésima variable en el $l$-ésimo grupo se nota por $\bar{X}_{(l) j}, l=1, \ldots, k$ con $n_{(1)}$ igual al número de individuos en el $l$-ésimo conglomerado. Según Díaz (2012), lo que se busca con la aplicación del algoritmo k-medias es obtener grupos con la menor variabilidad posible, por lo anterior, sugiere las siguientes estrategias: 1 ) seleccionar los primeros $K$ elementos de la muestra como los $K$ grupos iniciales de vectores de medias y $K$ los elementos más alejados; 2 ) iniciar con un valor de $K$ tan grande como sea necesario, y proceder a formar centroides de los grupos alejados a un múltiplo de la desviación estándar sobre cada variable; 3 ) nombrar los elementos de 1 a $n$ y escoger los que resulten marcados con los números $n / k, 2 n / k, \ldots,(k-1) / k$ y $n$ y 4$)$ seleccionar $K$ y la configuración inicial de los grupos por el conocimiento previo del problema.

\section{Resultados}

La metodología de clasificación del algoritmo no jerárquico k-medias permite procesar un número ilimitado de datos, pero se debe conocer previamente el número de grupos que se desea obtener (Díaz, 2012). Se recurrió a técnicas estadísticas para conocer el número de grupos, como lo son el Codo de Elbow y el Coeficiente de Silueta o Silhouette. Antes de aplicar las técnicas que se relacionan a continuación se requirió estandarizar los datos debido a que la variable número de hectáreas tenía mayor variabilidad que las variables de conteo de delitos.

El Codo de Elbow. Esta técnica identifica el número de grupos en el punto donde la inercia existente de un grupo a otro se reduce de manera significativa o brusca, y permite ver una curva pronunciada parecida a un codo como el que resulta en la figura 1. 


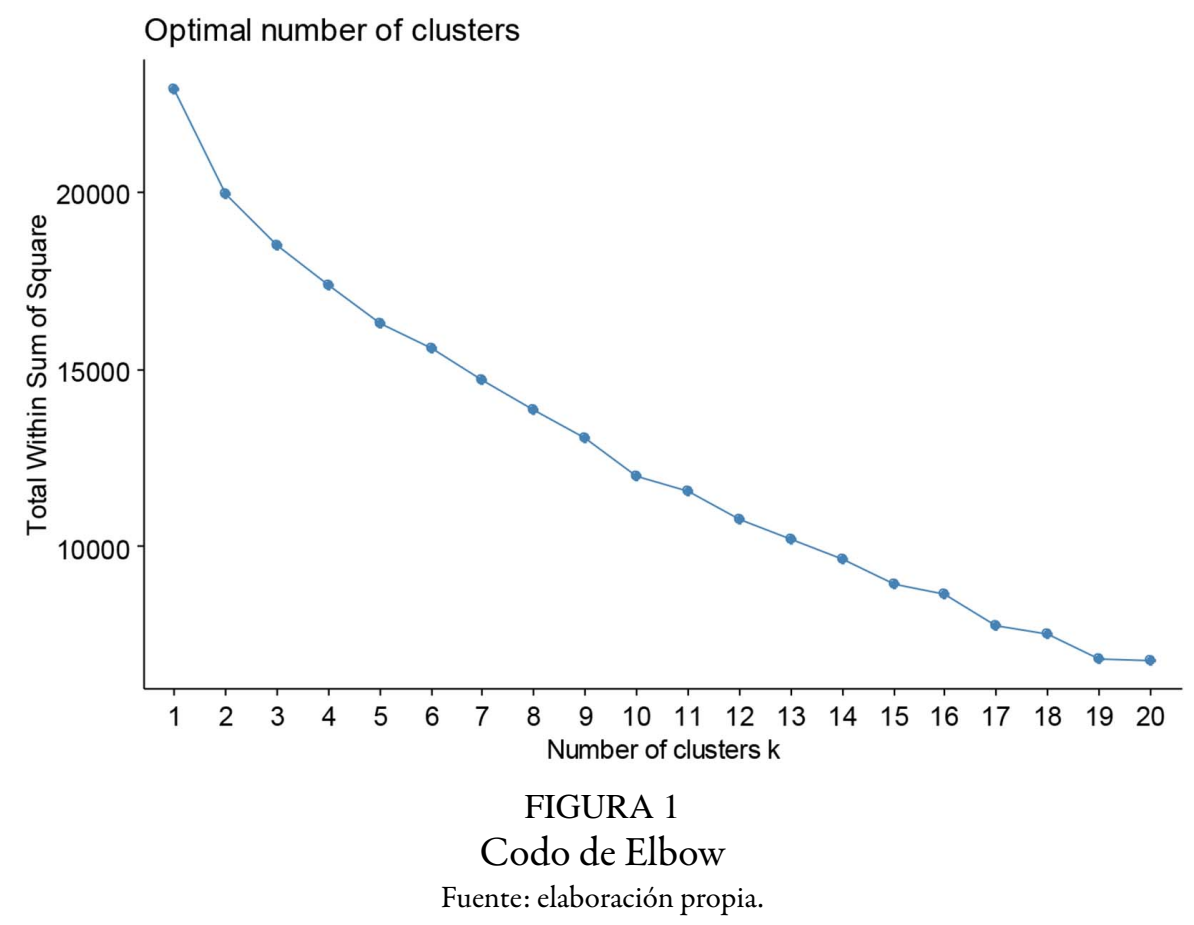

En la figura 1 no es muy clara la información respecto al número de clústeres, aunque se logra visualizar una mayor separación entre la partición de dos y tres clusters, lo cual es un indicio para seleccionar tres grupos en la partición. Se realizó un análisis del coeficiente de silueta para corroborar la partición adecuada.

El coeficiente de Silueta o Silhouette. En esta técnica el número de grupos óptimo se encuentra cuando el valor del coeficiente se aproxima a la unidad, esto se debe a que un índice cercano a uno (1) indica un buen agrupamiento; cercano a cero (0) el agrupamiento es indiferente y en el caso que se aproxime a menos uno $(-1)$, indica que existe un mal agrupamiento. Ver figura 2.

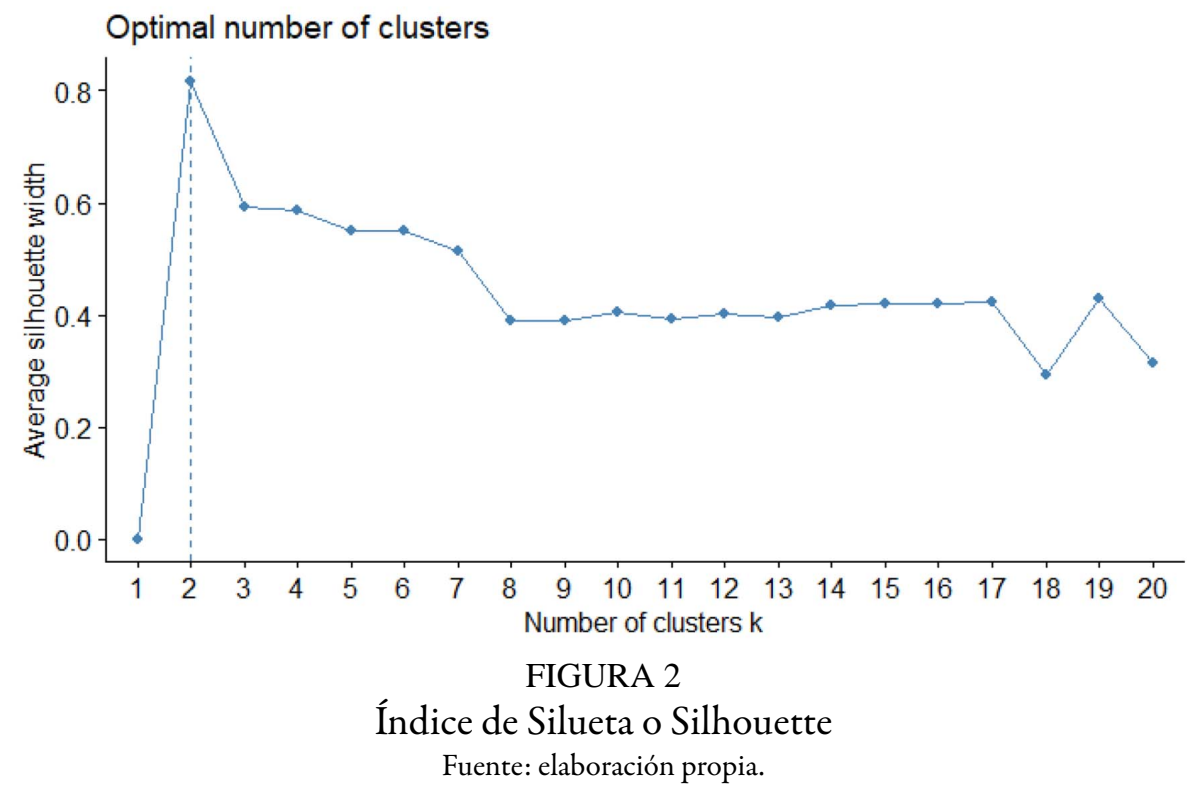

Según el índice de Silhouette lo óptimo son dos grupos debido a que este punto es el que más se aproxima a uno, no obstante, se podría agrupar en tres, cuatro y hasta cinco clústeres debido a que el índice se mantiene alrededor del 0,5 para estas divisiones. Teniendo en cuenta los resultados del Codo de Elbow y del índice de 
Silueta se opta por aplicar una clasificación de tres grupos, con el fin de alcanzar la clasificación deseada bajo este algoritmo, y así lograr calificar los municipios de Colombia en un nivel de riesgo bajo, medio y alto.

\section{Grupos obtenidos en la Aplicación del Algoritmo K-Medias}

Una vez aplicado el algoritmo K-Medias para la agrupación de los municipios de Colombia basados en la cantidad de delitos materializados y relacionados con los delitos fuente de LAFT se obtuvo la agrupación que se presenta en la tabla 2.

TABLA 2

\begin{tabular}{|c|c|c|c|}
\hline Conglomerado & Número de municipios & $\begin{array}{l}\text { Porcentaje } \\
(\%)\end{array}$ & $\begin{array}{c}\text { Acumulado } \\
(\%)\end{array}$ \\
\hline 1 & 924 & 84,62 & 84,62 \\
\hline 2 & 159 & 14,56 & 99,18 \\
\hline 3 & 9 & 0,82 & 100,00 \\
\hline Total & 1.092 & 100,00 & \\
\hline
\end{tabular}

Fuente: elaboración propia.

Del total de los 1.092 municipios de Colombia, se observa en la tabla 2, que el 84,62\% (924) se encuentran agrupados en el clúster uno; el clúster dos contiene el 14,56\% (159) y por último, encontramos el clúster tres el cual contiene el 0,82\% (9) de los municipios en Colombia.

En la figura 3 se expresa gráficamente la distribución de los municipios en cada uno de los conglomerados calculados en un plano factorial de dos dimensiones de un análisis de componentes principales usado exclusivamente a manera representativa para comprender la distancia de los municipios entre sí.

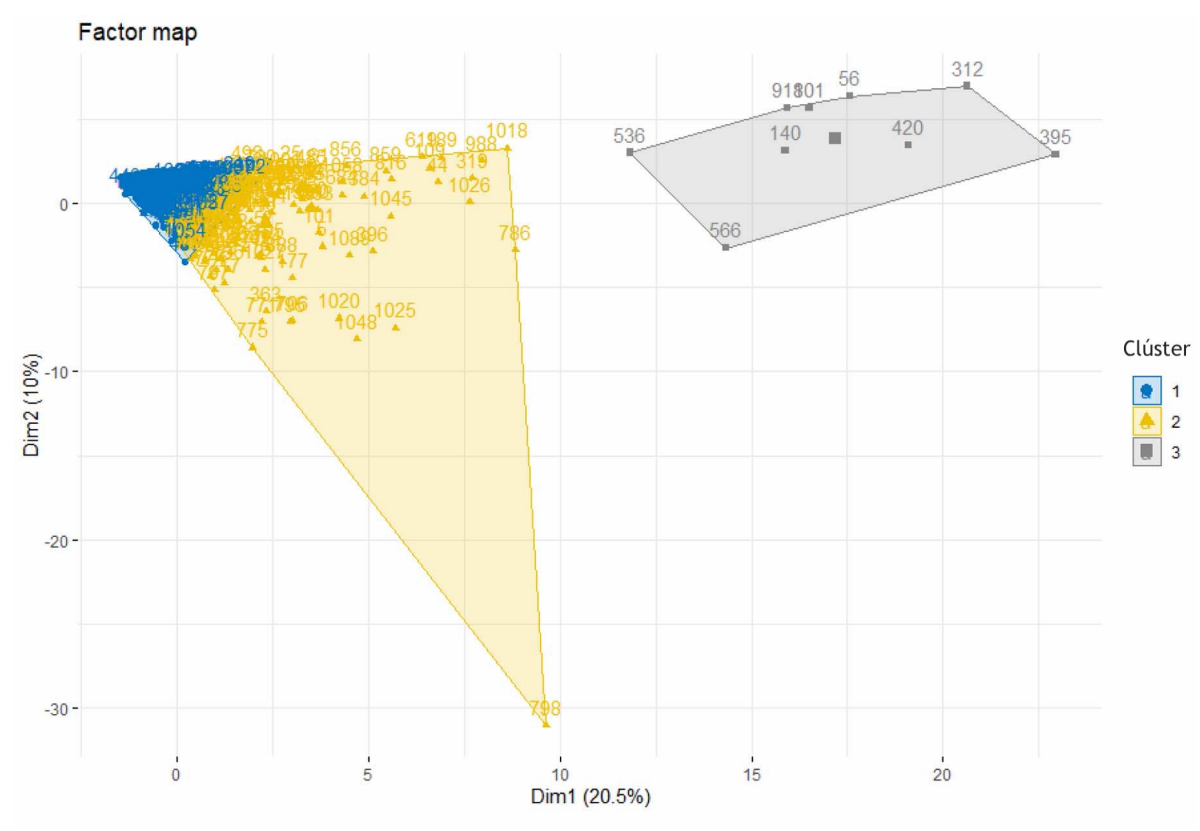

FIGURA 3

Agrupación de municipios Algoritmo K-Medias

Fuente: elaboración propia. 


\section{Agrupación propuesta}

Para proceder con la descripción de los conglomerados obtenidos, se recurre a la gráfica de coordenadas paralelas, en el cual se puede observar el comportamiento de cada variable. En la figura 4 se pueden observar las medias de cada una de las variables y, por tanto, el comportamiento de cada clúster. A través de esta figura se puede visualizar que el conglomerado uno presenta para cada una de las variables medias con valores cercanos al cero; lo cual permite deducir y nombrar a este conglomerado como Municipios de Bajo Riesgo en Materialización de delitos Fuente de LAFT.

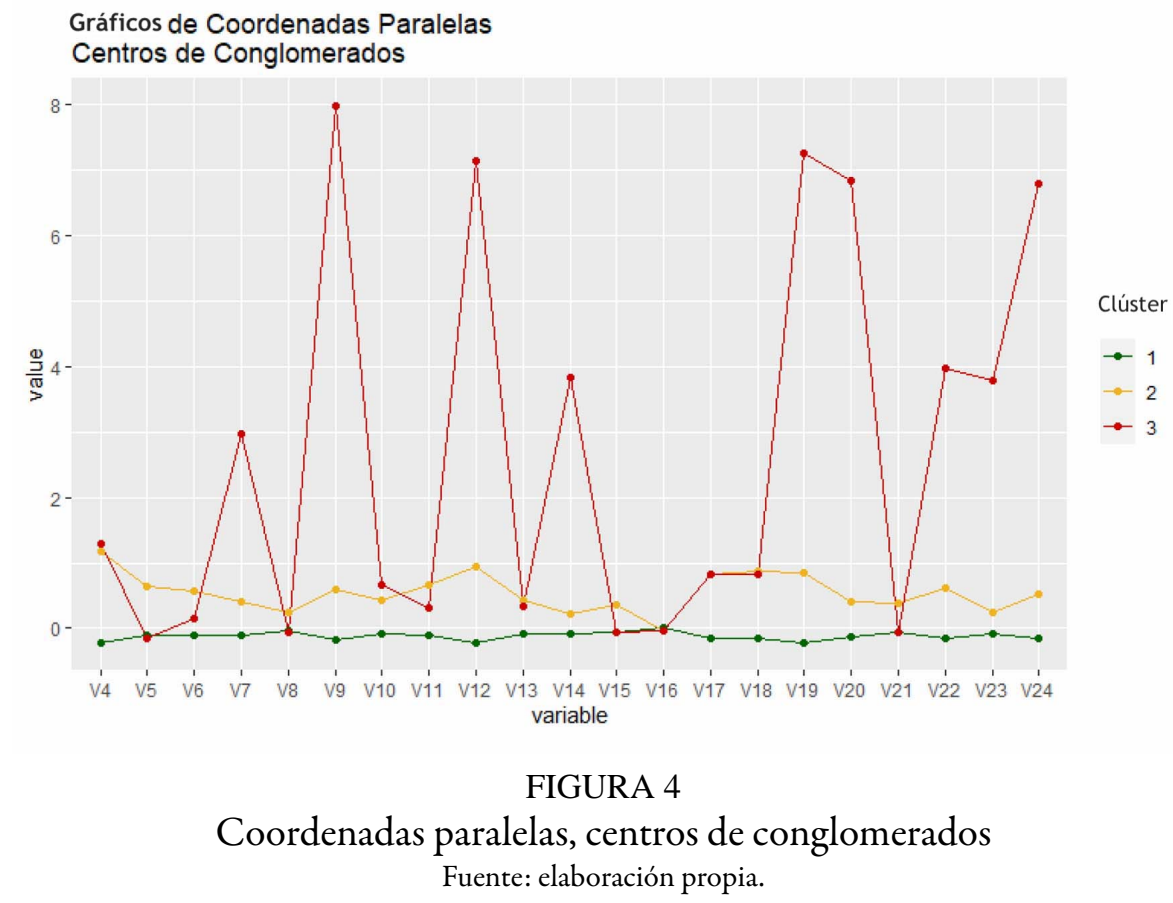

Por otra parte, al analizar el comportamiento del conglomerado 2 se puede ver que los valores de los centros de este grupo se encuentran un poco más retirados del cero y no sobrepasan los centroides del conglomerado tres. Se puede concluir que este conglomerado corresponde a los Municipios con Riesgo Medio en Materialización de delitos Fuente de LAFT.

Finalmente, en el tercer conglomerado se puede advertir que los valores de los centroides para la gran mayoría de variables son superiores a los de los dos grupos anteriores; por consiguiente, este grupo se puede nombrar como Municipios con Riesgo Alto en Materialización de delitos Fuente de LAFT.

Teniendo en cuenta los diferentes conglomerados obtenidos en la aplicación del algoritmo K-Medias, en la figura 5 visualiza la clasificación obtenida en un mapa de Colombia. 


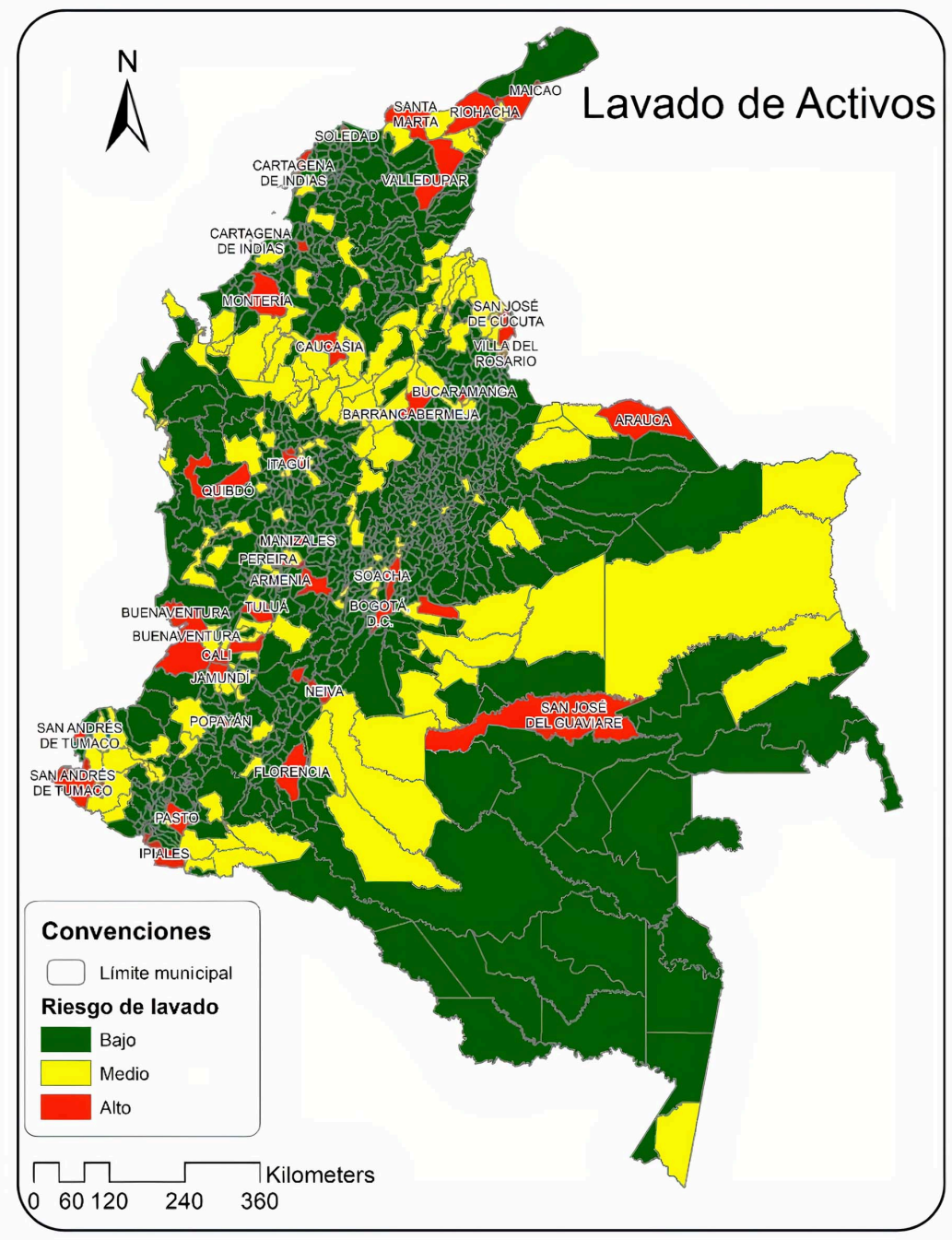

FIGURA 5

Clasificación de los municipios de Colombia, según el Nivel de riesgo frente a los delitos fuente de LAFT Fuente: elaboración propia.

El mapa permite conocer de forma georreferencial la clasificación según el nivel de riesgo identificado para cada municipio. El color verde hace referencia a aquellos municipios clasificados como Nivel de Riesgo Bajo o también llamado conglomerado uno (1). El color amarillo está relacionado con los municipios clasificados como Nivel de Riesgo Medio o conglomerado dos (2). Por último, los municipios pertenecientes al color rojo son clasificados como Nivel de Riesgo Alto o conglomerado tres (3). Estos niveles de riesgo están relacionados a los delitos fuente del LAFT. En el anexo tabla A2 se encuentra el listado de los nombres de todos los municipios según la clasificación propuesta.

De la figura 5 se puede concluir que los principales municipios fronterizos de Colombia están agrupados en el Nivel de Riesgo Alto -exceptuando a Leticia- lo mismo que los principales municipios colombianos, desde el punto de vista de desarrollo económico y densidad poblacional. No obstante, el color predominante en esta figura es el verde, lo que indica desde el punto de vista geográfico que Colombia puede ser clasificada como nivel de riesgo bajo frente a los delitos fuente de LAFT. Esta clasificación es válida dentro de Colombia, pero podría cambiar al hacer la comparación con ciudades de otros países. 


\section{Conclusiones}

De acuerdo con los resultados, tan sólo 39 municipios presentan un riesgo alto en delitos fuentes LAFT, estos se caracterizan por ser fronterizos y tener un alto número de habitantes y de flujo comercial. Mientras que 159 municipios presentan un riesgo medio, estos tal vez, son los municipios que más información nueva aporta a este tema, pues no siempre son municipios fronterizos ni con altos flujo comercial pero sus niveles de delitos asociados al lavado los ponen en alerta para las entidades financieras y cooperativas que quieren operar en esas regiones, por otro lado hay 924 municipios con un riesgo bajo, los cuales son municipios con bajos índices de violencia y pocos poblados relativos a su tamaño. El porcentaje de participación de aquellos municipios con un riesgo alto de este tipo de delitos es menor que el $1 \%$ y da una señal positiva sobre las medidas que se han llevado a cabo para controlar este delito.

Pese a las medidas judiciales, los reportes de delitos fuente LAFT en 30 de los municipios de alto riesgo sobrepasan la cifra de 550 reportes en 2019. Estas zonas, previamente clasificadas en este grupo, deben ser atendidas rápidamente y las entidades financieras y judiciales deben estar muy atentas a la dinámica financiera y de mercado que se presenta en estos territorios. En el caso del conglomerado de riesgo bajo (Clúster 1), las entidades financieras podrían establecer negocios en estos municipios sin tener que hacer una inversión en prevención tan elevada como en municipios de alto riesgo, implementando algunas medidas de control para asegurar la operación teniendo en cuenta la situación política y social de cada lugar, que pueda afectar el desarrollo de la actividad financiera, dando cumplimiento a la normatividad colombiana frente al riesgo de LAFT.

Es preciso mencionar que los hallazgos de esta investigación permiten medir el riesgo LAFT (mediante el factor Jurisdicción) a través de una herramienta a bajo costo que le otorga a las empresas la posibilidad de identificar los municipios con mayor afluencia de delitos fuente para el LAFT y sobre ella guiar las decisiones sobre apertura de nuevas sucursales y/o corresponsales.

$\mathrm{Al}$ realizar esta clasificación se pueden tomar medidas más específicas respecto a la prevención que deben realizar las entidades financieras y generar un enfoque más especializado en cuanto al seguimiento del riesgo del lavado de activos en los municipios. Si se adoptan las políticas y soluciones necesarias, el riesgo alto y medio pueden enfocar la inversión en prevención y así los delitos fuentes LAFT podrían reducirse de forma significativa.

\section{Declaración de los autores y agradecimientos}

Este documento es parte del proyecto de grado de la especialización en estadística Clasificación del nivel de riesgo de los municipios colombianos mediante la aplicación del análisis multivariado, teniendo en cuenta la ocurrencia de delitos fuente de lavado de activos y financiación del terrorismo en el 2019 de la escuela de matemáticas de Universidad Industrial de Santander y contó con la cooperación de personal de la Financiera Comultrasan. Agradecemos a María Camila Gómez Pradilla y a Jeferson Andrés Fuentes Gonzáles sus aportes en la mejora de este documento.

\section{Consideraciones éticas}

Los autores declaran que la investigación no requirió de un aval ético. 


\section{Contribución de los autores}

Henry Sebastián Rangel: diseño metodológico, procesamiento de datos, construcción de resultados y escritura del documento. Gilma Barrera: planteamiento del problema, revisión de literatura, definición y búsqueda de variables y escritura del documento. Oscar Mauricio Gómez: planteamiento del problema, revisión de literatura, definición y búsqueda de variables y escritura del documento.

\section{Financiación}

Esta investigación no contó con financiación.

\section{Conflictos de interés}

Los autores declarar no tener conflicto de interés asociados al desarrollo de esta investigación.

\section{Referencias}

Albanese, D. E. (2012). Análisis y evaluación de riesgos: aplicación de una matriz de riesgo en el marco de un plan de prevención contra el lavado de activos. Revista Base (Administração e Contabilidade) da UNISINOS, 9(3), 206-215. https://www.redalyc.org/articulo.oa?id=337228651001

Bagley, B. M. (2000). Narcotráfico, violencia política y política exterior de estados Unidos hacia Colombia en los noventa. Colombia Internacional (49-50), 5-38. https://doi.org/10.7440/colombiaint49-50.2000.01

Bayona-Rodríguez, H. (2019). Money laundering in rural areas with illicit crops: Empirical evidence for Colombia. Crime, Law and Social Change, 72, 387-417. https://doi.org/10.1007/s10611-019-09822-z

Díaz, A. M., \& Sanchez, F. (2004). Geografía de los cultivos ilícitos y conflicto armado en Colombia. Documento CEDE, 2004-18.

Diaz, L. (2012). Análisis estadístico de datos multivariados. Bogotá, Publicaciones. Universidad Nacional de Colombia.

Fiscalía General de la Nación (2021). Conteo de procesos [base de datos conteo de procesos]. Repositorio. https://d ev.socrata.com/

Gobierno Nacional (2016, 24 de noviembre). Acuerdo final para la terminación del conflicto y la construcción de una paz estable y duradera. http://www.centrodememoriahistorica.gov.co/descargas/finAcuerdoPazAgosto2016/1 2-11-2016-Nuevo-Acuerdo-Final.pdf

Indepaz $(2021,5,2)$. Informe sobre grupos armados ilegales, Colombia, 2017-2018. Recuperado el 28 de febrero de 2020 de http://www.indepaz.org.co/conflictos-armados-focalizados/

Levi, M. (2020). Evaluating the control of money laundering and its underlying offences: the search for meaningful data. Asian Journal of Criminology, 15, 301-320. https://doi.org/10.1007/s11417-020-09319-y

Levi, M., \& Reuter, P. (2006). Money Laundering. Crime and Justice, 34(1), 289-375. https://doi.org/10.1086/501 508

Lozano, A. (2008). El perfil financiero: una estrategia para detectar el lavado de activos. Revista Criminalidad, 50(2), 43-55. http://www.scielo.org.co/pdf/crim/v50n2/v50n2a04.pdf

MinJusticia (2015). Informe Ejecutivo resultados evaluación nacional de riesgo de lavado de activos y financiación del terrorismo. Bogotá: Observatorio de Drogas de Colombia. http://www.odc.gov.co/Portals/1/publicaciones/pd f/delitos-relacionados-drogas/CR1032015_informe_evaluacion_riego_lavado_activos_2015.pdf 
Observatorio de Drogas de Colombia (2021, 5, 2). Número de Hectáreas con presencia de Cultivos Ilícitos en 2019. Recuperado el 28 de febrero de 2020 de http://www.odc.gov.co/sidco/oferta/cultivos-ilicitos/departamento-m unicipio

Peña, D. (2002). Análisis de datos multivariantes. Madrid. McGraw-Hill.

Puyana, G. (1999). Omisión de control de lavado de activos: Un delito con tipicidad defectuosa. Derecho Penal y Criminologia, 21(67), 51-78. https://revistas.uexternado.edu.co/index.php/derpen/article/view/1135

Rocha, R. (2001). El narcotráfico y la economía de Colombia: Una mirada a las políticas. Planeación y Desarrollo, XXXII(3), 427-470. https://colaboracion.dnp.gov.co/CDT/RevistaPD/2001/pd_vXXXII_n3_2001_art.3.p df

Rocha, R. (2014). La riqueza del narcotráfico y la desigualdad en Colombia, 1976-2012. Revista Criminalidad, 56(2), 273-290. http://www.scielo.org.co/pdf/crim/v56n2/v56n2a07.pdf

Rocha-Salazar, J., Segovia-Vargas, M., \& Camacho-Miñano, M. (2021). Money laundering and terrorism financing detection using neural networks and an abnormality indicator. Expert Systems with Applications, 169(4). https: //doi.org/10.1016/j.eswa.2020.114470

Rodríguez, J. P., \& Castro, R. M. (2020). Evaluación del modelo de Colombia sobre el riesgo de lavado de activos y financiación del terrorismo. En H. A. Hernández, La eficacia de las normas de prevención, detección y sanción del lavado de activos en Colombia (pp. 91-105). Ibagué: Ediciones Unibagué. https://doi.org/10.35707/97895875 4330804

Romaniuk, P. (2014). The state of the art on the financing of terrorism. The RUSI Journal, 159(2), 6-17. https://doi .org/10.1080/03071847.2014.912794

Sintura, F. J. (2010). La prevención del lavado de activos como una política de buen gobierno corporativo, necesaria para evitar los efectos de la Lista Clinton en Colombia. Revista Internacional Derecho Penal Contemporáneo, (32), 5-40.http://legal.legis.com.co/document/Index?obra=rpenal\&document=rpenal_8fd557db5fa8d098e0 $430 \mathrm{a} 010151 \mathrm{~d} 098$

UIAF (2019). Informe Ejecutivo: Evaluación Nacional de Riesgo. Bogotá: Unidad de Información y Análisis Financiero. https://www.uiaf.gov.co/sistema_nacional_ala_cft/evaluaciones_nacionales_riesgo/evaluacion_na cional_riesgo_2019

Ulloa, L. (2018). Marco jurídico del lavado de activos y de la captación masiva habitual de dineros desde un enfoque de Derecho Administrativo. Via inveniendi et iudicandi, 13(2), 81-106. https://doi.org/10.15332/s1909-0528 .2018.0002.05 
Henry Sebastián Rangel Quiñonez a, et al. Clasificación del riesgo de lavado de activos y f...

\section{Anexos}

TABLA A1

Código $\mathrm{R}$ aplicado para análisis de conglomerados

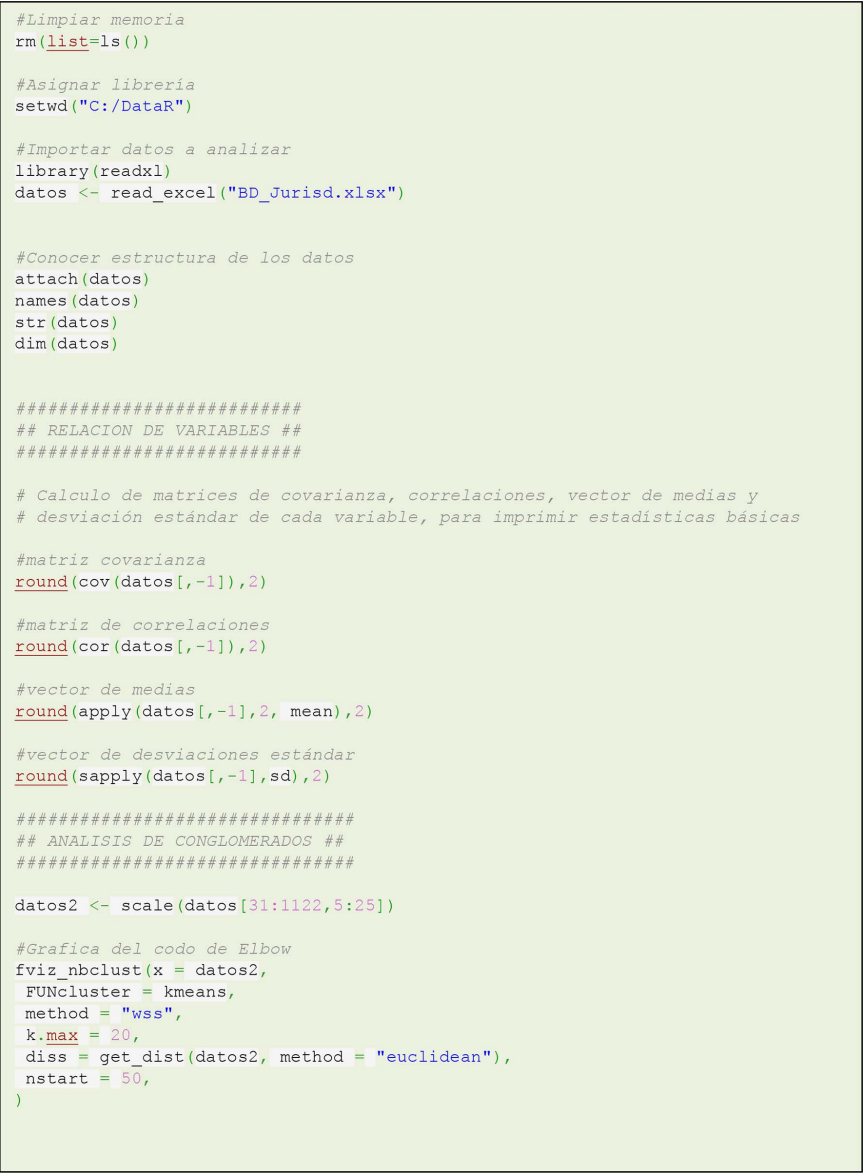

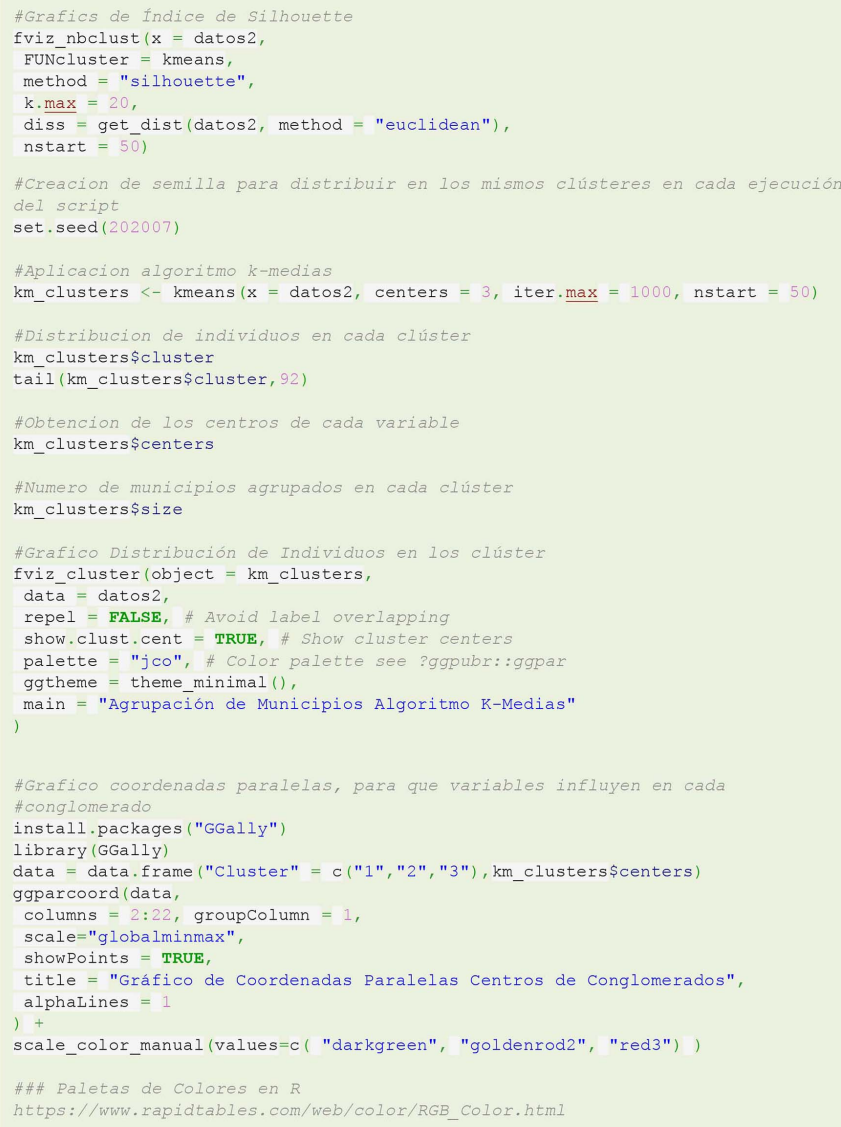

Fuente: elaboración propia. 
TABLA A2

Clasificación de municipios en cada conglomerado

\begin{tabular}{|c|c|c|}
\hline \multicolumn{3}{|c|}{ CONGLOMERADO UNO (1) } \\
\hline Municipio & Municipio & Municipio \\
\hline Abejorral (Antioquia) & Santa Bárbara (Antioquia) & Turbana (Bolívar) \\
\hline Abriaqui (Antioquia) & Santa Rosa De Osos (Antioquia) & Villanueva (Bolivar) \\
\hline Alejandría (Antioquia) & El Santuario (Antioquia) & Zambrano (Bolívar) \\
\hline Andes (Antioquia) & Sonsón (Antioquia) & Almeida (Boyacá) \\
\hline Angelópolis (Antioquia) & Sopetrán (Antioquia) & Aquitania (Boyacá) \\
\hline Angostura (Antioquia) & Támesis (Antioquia) & Arcabuco (Boyacá) \\
\hline Anzá (Antioquia) & Tarso (Antioquia) & Belén (Boyacá) \\
\hline Arboletes (Antioquia) & Titiribi (Antioquia) & Berbeo (Boyacá) \\
\hline Argelia (Antioquia) & Toledo (Antioquia) & Beteitiva (Boyacá) \\
\hline Armenia (Antioquia) & Uramita (Antioquia) & Boavita (Boyacá) \\
\hline Belmira (Antioquia) & Valparaiso (Antioquia) & Boyacá (Boyacá) \\
\hline Betania (Antioquia) & Venecia (Antioquia) & Briceño (Boyacá) \\
\hline Betulia (Antioquia) & Vigia Del Fuerte (Antioquia) & Buenavista (Boyacá) \\
\hline Buriticá (Antioquia) & Yali (Antioquia) & Busbanza (Boyacá) \\
\hline Caicedo (Antioquia) & Yolombo (Anti & Caldas (Boyacá) \\
\hline Campamento (Antioquia) & Baranoa (Atlántico) & Campohermoso (Boyacá) \\
\hline Caracoli (Antioquia) & Campo De La Cruz (Atlántico) & Cerinza (Boyacá) \\
\hline Caramanta (Antioquia) & Candelaria (Atlántico) & Chinavita (Boyacá) \\
\hline Carolina (Antioquia) & Galapa (Atlántico) & Chiquinquirá (Boyacá) \\
\hline Chigorodó (Antioquia) & Juan De Acosta (Atlántico) & Chiscas (Boyacá) \\
\hline Cisneros (Antioquia) & Luruaco (Atlántico) & Chita (Boyacá) \\
\hline Cocomá (Antioquia) & Manatí (Atlántico) & Chitaraque (Boyacá) \\
\hline Concepción (Antioquia) & Palmar De Varela (Atlántico) & Chivata (Boyacá) \\
\hline Concordia (Antioquia) & Piojó(Atlántico) & Ciénega (Boyacá) \\
\hline Copacabana (Antioquia) & Polonuevo (Atlántico) & Cómbita (Boyacá) \\
\hline Dabeiba (Antioquia) & Ponedera (Atlántico) & Coper (Boyacá) \\
\hline Donmatias(Antioquia) & Puerto Colombia (Atlántico) & Corrales (Boyacá) \\
\hline Ebéjico (Antioquia) & Repelón (Atlántico) & Covarachía(Boyacá) \\
\hline Entrerrios (Antioquia) & Sabanagrande (Atlántico) & Cubará(Boyacá) \\
\hline Fredonia (Antioquia) & Sabanalarga (Atlántico) & Cucaita (Boyacá) \\
\hline Frontino (Antioquia) & Santa Lucía (Atlántico) & Cuitiva (Boyacá) \\
\hline Giraldo (Antioquia) & Santo Tomás(Atlántico) & Chíquiza (Boyacá) \\
\hline Gómez Plata (Antioquia) & Suan (Atlántico) & Chivor (Boyacá) \\
\hline Granada (Antioquia) & Tubara (Atlántico) & El Cocuy (Boyacá) \\
\hline Guadalupe (Antioquia) & Usiacuri (Atlántico) & E1 Espino (Boyacá) \\
\hline Guatapé (Antioquia) & Achí (Bolívar) & Firavitoba (Boyacá) \\
\hline Heliconia (Antioquia) & Altos Del Rosario (Bolivar) & Floresta (Boyacá) \\
\hline Hispania (Antioquia) & Arenal (Bolivar) & Gachantivá (Boyacá) \\
\hline Jardín (Antioquia) & Arroyohondo (Bolivar) & Gámeza (Boyacá) \\
\hline Jericó (Antioquia) & Barranco De Loba (Bolivar) & Garagoa (Boyacá) \\
\hline La Ceja (Antioquia) & Calamar (Bolivar) & Guacamayas (Boyacá) \\
\hline La Pintada (Antioquia) & Cantagallo (Bolívar) & Guateque (Boyacá) \\
\hline La Unión (Antioquia) & Cicuco (Bolivar) & Guayatá(Boyacá) \\
\hline Liborina (Antioquia) & Córdoba (Bolívar) & Güicán (Boyacá) \\
\hline Maceo (Antioquia) & Clemencia (Bolívar) & Iza (Boyacá) \\
\hline Montebello (Antioquia) & El Guamo (Bolívar) & Jenesano (Boyacá) \\
\hline Murindó(Antioquia) & El Peñón (Bolívar) & Jericó (Boyacá) \\
\hline Mutatá (Antioquia) & Hatillo De Loba (Bolívar) & Labranzagrande (Boyacá) \\
\hline Nariño (Antioquia) & Mahates (Bolívar) & La Capilla (Boyacá) \\
\hline Necocli (Antioquia) & Margarita (Bolívar) & La Victoria (Boyacá) \\
\hline Olaya (Antioquia) & María La Baja (Bolivar) & La Uvita (Boyacá) \\
\hline Peñol (Antioquia) & Montecristo (Bolivar) & Villa De Leyva (Boyacá) \\
\hline Peque (Antioquia) & Mompós (Bolivar) & Macanal (Boyacá) \\
\hline Pueblorrico (Antioquia) & Norosí(Bolivar) & Maripí(Boyacá) \\
\hline Puerto Nare (Antioquia) & Pinillos (Bolivar) & Miraflores (Boyacá) \\
\hline Puerto Triunfo (Antioquia) & Regidor (Bolivar) & Mongua (Boyacá) \\
\hline Retiro (Antioquia) & Río Viejo (Bolivar) & Monguí (Boyacá) \\
\hline Sabanalarga (Antioquia) & San Cristóbal (Bolívar) & Moniquirá (Boyacá) \\
\hline Salgar (Antioquia) & San Estanislao (Bolivar) & Motavita (Boyacá) \\
\hline San Andrés De Cuerquia (Antioquia) & San Fernando (Bolivar) & Muzo (Boyacá) \\
\hline San Carlos (Antioquia) & San Jacinto (Bolivar) & Nobsa (Boyacá) \\
\hline San Francisco (Antioquia) & San Jacinto Del Cauca (Bolivar) & Nuevo Colón (Boyacá) \\
\hline San Jerónimo (Antioquia) & San Juan Nepomuceno (Bolivar) & Oicata (Boyacá) \\
\hline San José De La Montaña (Antioquia) & San Martín De Loba (Bolivar) & Otanche (Boyacá) \\
\hline San Juan De Urabá (Antioquia) & Santa Catalina (Bolivar) & Pachavita (Boyacá) \\
\hline San Luis (Antioquia) & Santa Rosa (Bolivar) & Páez (Boyacá) \\
\hline San Pedro de los Milagros (Antioquia) & Simití(Bolívar) & Paipa (Boyacá) \\
\hline San Pedro De Urabá (Antioquia) & Soplaviento (Bolívar) & Pajarito (Boyacá) \\
\hline San Rafael (Antioquia) & Talaigua Nuevo (Bolivar) & Panqueba (Boyacá) \\
\hline San Roque (Antioquia) & Tiquisio (Bolivar) & Pauna (Boyacá) \\
\hline San Vicente (Antioquia) & Turbaco (Bolivar) & Paya (Boyacá) \\
\hline Paz De Río (Boyacá) & Viterbo (Caldas) & Cotorra (Córdoba) \\
\hline Pesca (Boyacá) & Albania (Caquetá) & La Apartada (Córdoba) \\
\hline Pisba (Boyacá) & Belén De Los Andaquíes(Caquetá) & Los Córdobas (Córdoba) \\
\hline Quipama (Boy & Curillo (Caquetá) & Momil (Córdoba) \\
\hline Ramiriquí (Boyacá) & El Doncello (Caquetá) & Moñitos (Córdoba) \\
\hline
\end{tabular}




\begin{tabular}{|c|c|c|}
\hline \multicolumn{3}{|c|}{ CONGLOMERADO UNO (1) } \\
\hline Municipio & Municipio & Municipio \\
\hline Ráquira (Boyacá) & El Paujil (Caquetá) & Pueblo Nuevo (Córdoba) \\
\hline Rondón (Boyacá) & La Montañita (Caquetá) & Puerto Escondido (Córdoba) \\
\hline Saboya (Boyacá) & Milán (Caquetá) & Purísima (Córdoba) \\
\hline Sáchica (Boyacá) & Morelia (Caquetá) & San Andrés Sotavento (Córdoba) \\
\hline Samacá (Boyacá) & San José Del Fragua (Caquetá) & San Antero (Córdoba) \\
\hline San Eduardo (Boyacá) & Solano (Caquetá) & San Bernardo del Viento (Córdoba) \\
\hline San José de Pare (Boyacá) & Solita (Caquetá) & San Carlos (Córdoba) \\
\hline San Luis de Gaceno (Boyacá) & Valparaíso (Caquetá) & San José de Ure (Córdoba) \\
\hline San Mateo (Boyacá) & Almaguer (Cauca) & San Pelayo (Córdoba) \\
\hline San Miguel De Sema (Boyacá) & Argelia (Cauca) & Tuchin (Córdoba) \\
\hline San Pablo De Borbur (Boyacá) & Balboa (Cauca) & Valencia (Córdoba) \\
\hline Santana (Boyacá) & Bolivar (Cauca) & Agua De Dios (Cundinamarca) \\
\hline Santa María (Boyacá) & Cajibío (Cauca) & Alban (Cundinamarca) \\
\hline Santa Rosa De Viterbo (Boyacá) & Caldono (Cauca) & Anapoima (Cundinamarca) \\
\hline Santa Sofia (Boyacá) & Florencia (Cauca) & Anolaima (Cundinamarca) \\
\hline Sativanorte (Boyacá) & Guachené (Cauca) & Arbeláez (Cundinamarca) \\
\hline Sativasur (Boyacá) & Guapi (Cauca) & Beltrán (Cundinamarca) \\
\hline Siachoque (Boyacá) & Inza (Cauca) & Bituima (Cundinamarca) \\
\hline Soata (Boyacá) & La Sierra (Cauca) & Bojacá (Cundinamarca) \\
\hline Socotá (Boyacá) & La Vega (Cauca) & Cabrera (Cundinamarca) \\
\hline Socha (Boyacá) & López (Cauca) & Cachipay (Cundinamarca) \\
\hline Somondoco (Boyacá) & Mercaderes (Cauca) & Caparrapi (Cundinamarca) \\
\hline Sora (Boyacá) & Miranda (Cauca) & Cáqueza (Cundinamarca) \\
\hline Sotaquira (Boyacá) & Morales (Cauca) & Carmen De Carupa (Cund.) \\
\hline Soraca (Boyacá) & Padilla (Cauca) & Chaguani (Cundinamarca) \\
\hline Susacon (Boyacá) & Páez (Cauca) & Chipaque (Cundinamarca) \\
\hline Sutamarchan (Boyacá) & Piamonte (Cauca) & Choachí (Cundinamarca) \\
\hline Sutatenza (Boyacá) & Piendamo (Cauca) & Chocontá (Cundinamarca) \\
\hline Tasco (Boyacá) & Purace (Cauca) & Cogua (Cundinamarca) \\
\hline Tenza (Boyacá) & Rosas (Cauca) & Cota (Cundinamarca) \\
\hline Tibana (Boyacá) & San Sebastián (Cauca) & Cucunubá (Cundinamarca) \\
\hline Tibasosa (Boyacá) & Santa Rosa (Cauca) & El Colegio (Cundinamarca) \\
\hline Tinjacá (Boyacá) & Silvia (Cauca) & El Peñón (Cundinamarca) \\
\hline Tipacoque (Boyacá) & Sotará (Cauca) & El Rosal (Cundinamarca) \\
\hline Toca (Boyacá) & Suarez (Cauca) & Fomeque (Cundinamarca) \\
\hline Togüi (Boyacá) & Sucre (Cauca) & Fosca (Cundinamarca) \\
\hline Tópaga (Boyacá) & Timbio (Cauca) & Funza (Cundinamarca) \\
\hline Tota (Boyacá) & Timbiquí (Cauca) & Fúquene (Cundinamarca) \\
\hline Tununguá (Boyacá) & Toribio (Cauca) & Gachalá (Cundinamarca) \\
\hline Turmequé (Boyacá) & Totoro (Cauca) & Gachancipá (Cundinamarca) \\
\hline Tuta (Boyacá) & Villa Rica (Cauca) & Gachetá(Cundinamarca) \\
\hline Tutaza (Boyacá) & Agustín Codazzi (Cesar) & Gama (Cundinamarca) \\
\hline Úmbita (Boyacá) & Astrea (Cesar) & Granada (Cundinamarca) \\
\hline Ventaquemada (Boyacá) & Becerril (Cesar) & Guachetá (Cundinamarca) \\
\hline Viracacha (Boyacá) & Bosconia (Cesar) & Guasca (Cundinamarca) \\
\hline Zetaquira (Boyacá) & Chimichagua (Cesar) & Guataquí (Cundinamarca) \\
\hline Aguadas (Caldas) & Chiriguaná(Cesar) & Guatavita (Cundinamarca) \\
\hline Anserma (Caldas) & El Copey (Cesar) & Guayabal De Siquima (Cund.) \\
\hline Aranzazu (Caldas) & El Paso (Cesar) & Guayabetal (Cundinamarca) \\
\hline Belalcázar(Caldas) & Gamarra (Cesar) & Gutiérrez(Cundinamarca) \\
\hline Chinchiná (Caldas) & González (Cesar) & Jerusalén(Cundinamarca) \\
\hline Filadelfia (Caldas) & La Gloria (Cesar) & Junín (Cundinamarca) \\
\hline La Merced (Caldas) & La Jagua De Ibirico (Cesar) & La Calera (Cundinamarca) \\
\hline Manzanares (Caldas) & Manaure (Cesar) & La Palma (Cundinamarca) \\
\hline Marquetalia (Caldas) & Pueblo Bello (Cesar) & La Peña (Cundinamarca) \\
\hline Marulanda (Caldas) & Río De Oro (Cesar) & La Vega (Cundinamarca) \\
\hline Neira (Caldas) & La Paz (Cesar) & Lenguazaque (Cundinamarca) \\
\hline Norcasia (Caldas) & San Alberto (Cesar) & Macheta (Cundinamarca) \\
\hline Pácora (Caldas) & San Diego (Cesar) & Madrid (Cundinamarca) \\
\hline Palestina (Caldas) & San Martín (Cesar) & Manta (Cundinamarca) \\
\hline Pensilvania (Caldas) & Tamalameque (Cesar) & Medina (Cundinamarca) \\
\hline Risaralda (Caldas) & Ayapel (Córdoba) & Nariño (Cundinamarca) \\
\hline Salamina (Caldas) & Buenavista (Córdoba) & Nemocón (Cundinamarca) \\
\hline Samaná (Caldas) & Canalete (Córdoba) & Nilo (Cundinamarca) \\
\hline San José (Caldas) & Cereté(Córdoba) & Nimaima (Cundinamarca) \\
\hline Supía (Caldas) & Chima (Córdoba) & Nocaima (Cundinamarca) \\
\hline Victoria (Caldas) & Chinú(Córdoba) & Venecia (Cundinamarca) \\
\hline Villamaría (Caldas) & CiénagaDe Oro (Córdoba) & Pacho (Cundinamarca) \\
\hline Paime (Cundinamarca) & Algeciras (Huila) & El Calvario (Meta) \\
\hline Pandi (Cundinamarca) & Altamira (Huila) & El Castillo (Meta) \\
\hline Paratebueno (Cundinamarca) & Baraya (Huila) & El Dorado (Meta) \\
\hline Pasca (Cundinamarca) & Campoalegre (Huila) & Fuente De Oro (Meta) \\
\hline Puerto Salgar (Cundinamarca) & Colombia (Huila) & Guamal (Meta) \\
\hline Pulí (Cundinamarca) & Elías (Huila) & Mapiripán (Meta) \\
\hline Quebradanegra (Cundinamarca) & Garzón (Huila) & Mesetas (Meta) \\
\hline Quetame (Cundinan & Gigante (Huila) & Uribe (Meta) \\
\hline
\end{tabular}




\begin{tabular}{|c|c|c|}
\hline \multicolumn{3}{|c|}{ CONGLOMERADO UNO (1) } \\
\hline Municipio & Municipio & Municipio \\
\hline Quipile (Cundinamarca) & Guadalupe (Huila) & Lejanías (Meta) \\
\hline Apulo (Cundinamarca) & Hobo (Huila) & Puerto Concordia (Meta) \\
\hline Ricaurte (Cundinamarca) & Iquira (Huila) & Puerto Rico (Meta) \\
\hline San Antonio Del Tequendama (Cund.) & Isnos (Huila) & Restrepo (Meta) \\
\hline San Bernardo (Cundinamarca) & La Argentina (Huila) & San Carlos de Guaroa (Meta) \\
\hline San Cayetano (Cundinamarca) & Nátaga (Huila) & San Juan de Arama (Meta) \\
\hline San Francisco (Cundinamarca) & Oporapa (Huila) & San Juanito (Meta) \\
\hline San Juan De Rioseco (Cund.) & Paicol (Huila) & Albán (Nariño) \\
\hline Sasaima (Cundinamarca) & Palermo (Huila) & Aldana (Nariño) \\
\hline Sesquilé (Cundinamarca) & Palestina (Huila) & Ancuya (Nariño) \\
\hline Sibaté (Cundinamarca) & Pital (Huila) & Arboleda (Nariño) \\
\hline Simijaca (Cundinamarca) & Rivera (Huila) & Belén (Nariño) \\
\hline Sopó (Cundinamarca) & Saladoblanco (Huila) & Buesaco (Nariño) \\
\hline Subachoque (Cundinamarca) & San Agustín (Huila) & Colón (Nariño) \\
\hline Suesca (Cundinamarca) & Santa María (Huila) & Consaca (Nariño) \\
\hline Supatá (Cundinamarca) & Suaza (Huila) & Contadero (Nariño) \\
\hline Susa (Cundinamarca) & Tarqui (Huila) & Córdoba (Nariño) \\
\hline Sutatausa (Cundinamarca) & Tesalia (Huila) & Cuaspud (Nariño) \\
\hline Tabio (Cundinamarca) & Tello (Huila) & Cumbal (Nariño) \\
\hline Tausa (Cundinamarca) & Teruel (Huila) & Chachagüi (Nariño) \\
\hline Tena (Cundinamarca) & Timaná(Huila) & El Peñol (Nariño) \\
\hline Tenjo (Cundinamarca) & Villavieja (Huila) & El Rosario (Nariño) \\
\hline Tibacuy (Cundinamarca) & Yaguará(Huila) & El Tablón De Gomez (Nariño) \\
\hline Tibirita (Cundinamarca) & Barrancas (La Guajira) & El Tambo (Nariño) \\
\hline Tocaima (Cundinamarca) & El Molino (La Guajira) & Funes (Nariño) \\
\hline Tocancipá (Cundinamarca) & Fonseca (La Guajira) & Guachucal (Nariño) \\
\hline Topaipí (Cundinamarca) & Hatonuevo (La Guajira) & Guaitarilla (Nariño) \\
\hline Ubalá (Cundinamarca) & La Jagua Del Pilar (La Guajira) & Gualmatán (Nariño) \\
\hline Ubaque (Cundinamarca) & Manaure (La Guajira) & Iles (Nariño) \\
\hline Une (Cundinamarca) & Uribia (La Guajira) & Imues (Nariño) \\
\hline Útica (Cundinamarca) & Urumita (La Guajira) & La Cruz (Nariño) \\
\hline Vergara (Cundinamarca) & Villanueva (La Guajira) & La Florida (Nariño) \\
\hline Viani (Cundinamarca) & Algarrobo (Magdalena) & La Llanada (Nariño) \\
\hline Villagómez(Cundinamarca) & Aracataca (Magdalena) & La Tola (Nariño) \\
\hline Villapinzón (Cundinamarca) & Ariguaní(Magdalena) & La Unión (Nariño) \\
\hline Villeta (Cundinamarca) & Cerro San Antonio (Magdalena) & Leiva (Nariño) \\
\hline Viota (Cundinamarca) & Chivolo (Magdalena) & Linares (Nariño) \\
\hline Yacopí (Cundinamarca) & Concordia (Magdalena) & Los Andes (Nariño) \\
\hline Zipacón (Cundinamarca) & El Banco (Magdalena) & Mallama (Nariño) \\
\hline Alto Baudó (Chocó) & El Piñón(Magdalena) & Mosquera (Nariño) \\
\hline Atrato (Chocó) & El Retén (Magdalena) & Nariño (Nariño) \\
\hline Bagadó (Chocó) & Fundación (Magdalena) & Ospina (Nariño) \\
\hline Bajo Baudó (Chocó) & Guamal (Magdalena) & Francisco Pizarro (Nariño) \\
\hline Bojayá(Chocó) & Nueva Granada (Magdalena) & Potosi (Nariño) \\
\hline El Cantón del San Pablo (Chocó) & Pedraza (Magdalena) & Providencia (Nariño) \\
\hline Carmen del Daríen(Chocó) & Pijiño del Carmen (Magdalena) & Puerres (Nariño) \\
\hline Cértegui (Chocó) & Pivijay (Magdalena) & Pupiales (Nariño) \\
\hline Condoto (Chocó) & Plato (Magdalena) & Samaniego (Nariño) \\
\hline El Carmen De Atrato (Chocó) & Puebloviejo (Magdalena) & Sandona (Nariño) \\
\hline El Litoral Del San Juan (Chocó) & Remolino (Magdalena) & San Bernardo (Nariño) \\
\hline Lloro (Chocó) & Sabanas de San Ángel (Magdalena) & San Lorenzo (Nariño) \\
\hline Medio Atrato (Chocó) & Salamina (Magdalena) & San Pablo (Nariño) \\
\hline Medio Baudo (Chocó) & San Sebastián de Buenavista (Magdalena) & San Pedro De Cartago (Nariño) \\
\hline Medio San Juan (Chocó) & San Zenón (Magdalena) & Santa Bárbara (Nariño) \\
\hline Nuquí(Chocó) & Santa Ana (Magdalena) & Santacruz (Nariño) \\
\hline Río Iro (Chocó) & Santa Bárbara De Pinto (Magdalena) & Sapuyes (Nariño) \\
\hline Río Quito (Chocó) & Sitionuevo (Magdalena) & Taminango (Nariño) \\
\hline Riosucio (Chocó) & Tenerife (Magdalena) & Tuquerres (Nariño) \\
\hline San José del Palmar (Chocó) & Zapayán(Magdalena) & Yacuanquer (Nariño) \\
\hline Sipi (Chocó) & Zona Bananera (Magdalena) & Arboledas (Norte de Santander) \\
\hline Unguía (Chocó) & Barranca De Upía (Meta) & Bochalema (Norte de Santander) \\
\hline Unión Panamericana (Chocó) & Cabuyaro (Meta) & Bucarasica (Norte de Santander) \\
\hline Acevedo (Huila) & Castilla La Nueva (Meta) & Cácota (Norte de Santander) \\
\hline Agrado (Huila) & Cubarral (Meta) & Cáchira(Norte de Santander) \\
\hline Aipe (Huila) & Cumaral (Meta) & Chinacota (Norte de Santander) \\
\hline Chitaga (Norte de Santander) & Guadalupe (Santander) & Ambalema (Tolima) \\
\hline Cucutilla (Norte de Santander) & Guapotá (Santander) & Anzoátegui(Tolima) \\
\hline Durania (Norte de Santander) & Guavatá (Santander) & Armero (Tolima) \\
\hline Gramalote (Norte de Santander) & Güepsa (Santander) & Ataco (Tolima) \\
\hline Hacari (Norte de Santander) & Hato (Santander) & Cajamarca (Tolima) \\
\hline Herrán (Norte de Santander) & Jesus María (Santander) & Carmen de Apicalá (Tolima) \\
\hline Labateca (Norte de Santander) & Jordán (Santander) & Casabianca (Tolima) \\
\hline La Esperanza (Norte de Santander) & La Belleza (Santander) & Coello (Tolima) \\
\hline La Playa (Norte de Santander) & Landázuri(Santander) & Coyaima (Tolima) \\
\hline Lourdes (Norte de Santander) & La Paz (Santander) & Cunday (Tolima) \\
\hline Mutiscua (Norte de Santander) & Lebrija (Santander) & Dolores (Tolima) \\
\hline
\end{tabular}




\begin{tabular}{|c|c|c|}
\hline \multicolumn{3}{|c|}{ CONGLOMERADO UNO (1) } \\
\hline Municipio & Municipio & Municipio \\
\hline Pamplona (Norte de Santander) & Los Santos (Santander) & Falan (Tolima) \\
\hline Pamplonita (Norte de Santander) & Macaravita (Santander) & Flandes (Tolima) \\
\hline Puerto Santander (Norte de Santander) & Malagá(Santander) & Fresno (Tolima) \\
\hline Ragonvalia (Norte de Santander) & Matanza (Santander) & Guamo (Tolima) \\
\hline Salazar (Norte de Santander) & Mogotes (Santander) & Herveo (Tolima) \\
\hline San Calixto (Norte de Santander) & Molagavita (Santander) & Honda (Tolima) \\
\hline Santiago (Norte de Santander) & Ocamonte (Santander) & Icononzo (Tolima) \\
\hline Silos (Norte de Santander) & Oiba (Santander) & Lerida (Tolima) \\
\hline Toledo (Norte de Santander) & Onzaga (Santander) & Libano (Tolima) \\
\hline Villa Caro (Norte de Santander) & Palmar (Santander) & San Sebastian De Mariquita (Tolima) \\
\hline Buenavista (Quindío) & Palmas Del Socorro (Santander) & Melgar (Tolima) \\
\hline Circasia (Quindio) & Páramo (Santander) & Murillo (Tolima) \\
\hline Córdoba (Quindío) & Pinchote (Santander) & Natagaima (Tolima) \\
\hline Filandia (Quindío) & Puente Nacional (Santander) & Ortega (Tolima) \\
\hline Genova (Quindía) & Puerto Parra (Santander) & Palocabildo (Tolima) \\
\hline La Tebaida (Quindío) & Rionegro (Santander) & Piedras (Tolima) \\
\hline Montenegro (Quindío) & San Andrés (Santander) & Planadas (Tolima) \\
\hline Pijao (Quindío) & San Benito (Santander) & Prado (Tolima) \\
\hline Salento (Quindío) & San Gil (Santander) & Purificación(Tolima) \\
\hline Apia (Risaralda) & San Joaquín (Santander) & Rioblanco (Tolima) \\
\hline Balboa (Risaralda) & San Jose de Miranda (Santander) & Roncesvalles (Tolima) \\
\hline Belén De Umbria (Risaralda) & San Miguel (Santander) & Rovira (Tolima) \\
\hline Guatica (Risaralda) & San Vicente de Chucurí(Santander) & Saldaña (Tolima) \\
\hline La Celia (Risaralda) & Santa Bárbara(Santander) & San Antonio (Tolima) \\
\hline La Virginia (Risaralda) & Santa Helena Del Opón(Santander) & San Luis (Tolima) \\
\hline Marsella (Risaralda) & Simacota (Santander) & Santa Isabel (Tolima) \\
\hline Mistrato (Risaralda) & Socorro (Santander) & Suárez(Tolima) \\
\hline Pueblo Rico (Risaralda) & Suaita (Santander) & Valle De San Juan (Tolima) \\
\hline Quinchía (Risaralda) & Sucre (Santander) & Venadillo (Tolima) \\
\hline Santuario (Risaralda) & Suratá (Santander) & Villahermosa (Tolima) \\
\hline Aguada (Santander) & Tona (Santander) & Villarrica (Tolima) \\
\hline Albania (Santander) & Valle de San José (Santander) & Alcalá (Valle) \\
\hline Aratoca (Santander) & Vélez(Santander) & Andalucía (Valle) \\
\hline Barbosa (Santander) & Vetas (Santander) & Ansermanuevo (Valle) \\
\hline Barichara (Santander) & Villanueva (Santander) & Argelia (Valle) \\
\hline Betulia (Santander) & Zapatoca (Santander) & Bolívar (Valle) \\
\hline Bolivar (Santander) & Buenavista (Sucre) & Caicedonia (Valle) \\
\hline Cabrera (Santander) & Caimito (Sucre) & Calima (Valle) \\
\hline California (Santander) & Coloso (Sucre) & Candelaria (Valle) \\
\hline Capitanejo (Santander) & Corozal (Sucre) & ElÁguila(Valle) \\
\hline Carcasí(Santander) & Coveñas (Sucre) & El Cairo (Valle) \\
\hline Cepitá(Santander) & Chalán (Sucre) & El Dovio (Valle) \\
\hline Cerrito (Santander) & El Roble (Sucre) & Florida (Valle) \\
\hline Charalá (Santander) & Galeras (Sucre) & Ginebra (Valle) \\
\hline Charta (Santander) & Guaranda (Sucre) & La Cumbre (Valle) \\
\hline Chima (Santander) & La Unión (Sucre) & La Unión (Valle) \\
\hline Chipatá (Santander) & Los Palmitos (Sucre) & La Victoria (Valle) \\
\hline Concepción (Santander) & Morroa (Sucre) & Obando (Valle) \\
\hline Confines (Santander) & Ovejas (Sucre) & Restrepo (Valle) \\
\hline Contratación(Santander) & Palmito (Sucre) & Riofrío (Valle) \\
\hline Coromoro (Santander) & Sampués(Sucre) & Roldanillo (Valle) \\
\hline Curití (Santander) & San Benito Abad (Sucre) & San Pedro (Valle) \\
\hline El Carmen De Chucuri (Santander) & San Juan de Betulia (Sucre) & Sevilla (Valle) \\
\hline El Guacamayo (Santander) & San Marcos (Sucre) & Toro (Valle) \\
\hline El Peñón (Santander) & San Onofre (Sucre) & Trujillo (Valle) \\
\hline El Playón (Santander) & San Pedro (Sucre) & Ulloa (Valle) \\
\hline Encino (Santander) & San Luis de Since (Sucre) & Versalles (Valle) \\
\hline Enciso (Santander) & Sucre (Sucre) & Vijes (Valle) \\
\hline Florián (Santander) & Santiago de Tolú (Sucre) & Yotoco (Valle) \\
\hline Galán (Santander) & Tolú Viejo (Sucre) & Zarzal (Valle) \\
\hline Gámbita (Santander) & Alpujarra (Tolima) & Cravo Norte (Arauca) \\
\hline Guaca (Santander) & Alvarado (Tolima) & Puerto Rondón (Arauca) \\
\hline Chameza (Casanare) & San Francisco (Putumayo) & Cacahual (Guainía) \\
\hline Hato Corozal (Casanare) & San Miguel (Putumayo) & Pana Pana (Guainía) \\
\hline La Salina (Casanare) & Santiago (Putumayo) & Morichal (Guainía) \\
\hline Maní (Casanare) & Villagarzón (Putumayo) & Calamar (Guaviare) \\
\hline Monterrey (Casanare) & Providencia (San Andrés) & El Retorno (Guaviare) \\
\hline Nunchía (Casanare) & El Encanto (Amazonas) & Miraflores (Guaviare) \\
\hline Orocue (Casanare) & La Chorrera (Amazonas) & Mitú (Vaupés) \\
\hline Paz de Ariporo (Casanare) & La Pedrera (Amazonas) & Carurú (Vaupés) \\
\hline Pore (Casanare) & La Victoria (Amazonas) & Pacoa (Vaupés) \\
\hline Recetor (Casanare) & Miriti-Paraná (Amazonas) & Taraira (Vaupés) \\
\hline Sabanalarga (Casanare) & Puerto Alegría (Amazonas) & Papunaua (Vaupés) \\
\hline Sácama (Casanare) & Puerto Arica (Amazonas) & Yavaraté (Vaupés) \\
\hline San Luis de Palenque (Casanare) & Puerto Nariño (Amazonas) & La Primavera (Vichada) \\
\hline Támara (Casanare) & Puerto Santander (Amazonas) & Santa Rosalia (Vichada) \\
\hline
\end{tabular}




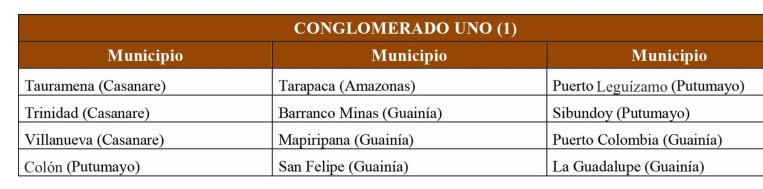

\begin{tabular}{|c|c|c|}
\hline \multicolumn{3}{|c|}{ CONGLOMERADO DOS (2) } \\
\hline Municipio & Municipio & Municipio \\
\hline Amaga (Antioquia) & Pailitas (Cesar) & Quimbaya (Quindio) \\
\hline Amalfi (Antioquia) & Pelaya (Cesar) & Dosquebradas (Risaralda) \\
\hline Anori (Antioquia) & Lorica (Córdoba) & Santa Rosa De Cabal (Risaralda) \\
\hline Santaféde Antioquia (Antioquia) & Montelíbano (Córdoba) & Cimitarra (Santander) \\
\hline Apartadó (Antioquia) & Planeta Rica (Córdoba) & Floridablanca (Santander) \\
\hline Barbosa (Antioquia) & Puerto Libertador (Córdoba) & Girón (Santander) \\
\hline Ciudad Bolivar (Antioquia) & Sahagún (Córdoba) & Piedecuesta (Santander) \\
\hline Briceño (Antioquia) & Tierralta (Córdoba) & Puerto Wilches (Santander) \\
\hline Cáceres (Antioquia) & Cajicá (Cundinamarca) & Sabana de Torres (Santander) \\
\hline Caldas (Antioquia) & Chía (Cundinamarca) & Majagual (Sucre) \\
\hline Cañasgordas (Antioquia) & Facatativá (Cundinamarca) & Chaparral (Tolima) \\
\hline Carepa (Antioquia) & Fusagasugáa (Cundinamarca) & Espinal (Tolima) \\
\hline El Carmen de Viboral (Antioquia) & Girardot (Cundinamarca) & Guadalajara De Buga (Valle) \\
\hline El Bagre (Antioquia) & Guaduas (Cundinamarca) & Bugalagrande (Valle) \\
\hline Envigado (Antioquia) & La Mesa (Cundinamarca) & Cartago (Valle) \\
\hline Girardota (Antioquia) & Mosquera (Cundinamarca) & Dagua (Valle) \\
\hline Guarne (Antioquia) & Silvania (Cundinamarca) & El Cerrito (Valle) \\
\hline Ituango (Antioquia) & Villa de San Diego de Ubaté (Cundinamarca) & Guacari (Valle) \\
\hline La Estrella (Antioquia) & Zipaquirá (Cundinamarca) & Pradera (Valle) \\
\hline Marinilla (Antioquia) & Acandi (Choco) & Yumbo (Valle) \\
\hline Nechi (Antioquia) & Bahia Solano (Choco) & Arauquita (Aranca) \\
\hline Puerto Berrio (Antioquia) & Istmina (Choco) & Fortul (Arauca) \\
\hline Remedios (Antioquia) & Jurado (Choco) & Saravena (Arauca) \\
\hline Sabaneta (Antioquia) & Novita (Choco) & Tame (Arauca) \\
\hline Santo Domingo (Antioquia) & Tado (Choco) & Yopal (Casanare) \\
\hline Segovia (Antioquia) & La Plata (Huila) & Aguazul (Casanare) \\
\hline Taraza (Antioquia) & Pitalito (Huila) & Mocoa (Putumayo) \\
\hline Turbo (Antioquia) & Albania (La Guajira) & Orito (Putumayo) \\
\hline Urrao (Antioquia) & Dibulla (La Guajira) & Puerto Asis (Putumayo) \\
\hline Valdivia (Antioquia) & Distracción (La Guajira) & Puerto Caicedo (Putumayo) \\
\hline Vegachi (Antioquia) & San Juan del Cesar (La Guajira) & Puerto Guzmán (Putumayo) \\
\hline Yarumal (Antioquia) & Ciénaga (Magdalena) & Valle Del Guamuez (Putumayo) \\
\hline Yondó (Antioquia) & Acacias (Meta) & San Andrés (San Andrés) \\
\hline Zaragoza (Antioquia) & Granada (Meta) & Leticia (Amazonas) \\
\hline Malambo (Atlántico) & La Macarena (Meta) & Inírida (Guainia) \\
\hline Arjona (Bolivar) & Puerto Gaitán (Meta) & Puerto Carreño (Vichada) \\
\hline El Carmen de Bolivar (Bolivar) & Puerto López (Meta) & Cumaribo (Vichada) \\
\hline Magangué (Bolivar) & Puerto Lleras (Meta) & Tibú (Norte de Santander) \\
\hline Morales (Bolivar) & San Martin (Meta) & Calarcá (Quindio) \\
\hline San Pablo (Bolivar) & Vistahermosa (Meta) & El Carmen (Norte de Santander) \\
\hline Santa Rosa del Sur (Bolivar) & Barbacoas (Nariño) & El Tarra (Norte de Santander) \\
\hline Tunja (Boyacá) & Cumbitara (Nariño) & El Zulia (Norte de Santander) \\
\hline Duitama (Boyacá) & El Charco (Nariño) & Los Patios (Norte de Santander) \\
\hline Puerto Boyacá (Boyacá) & Magüi (Nariño) & Ocaña (Norte de Santander) \\
\hline Sogamoso (Boyacá) & Olaya Herrera (Nariño) & San Cayetano (Norte de Santander) \\
\hline La Dorada (Caldas) & Policarpa (Nariño) & Sardinata (Norte de Santander) \\
\hline Marmato (Caldas) & Ricaurte (Nariño) & Teorama (Norte de Santander) \\
\hline Riosucio (Caldas) & Roberto Payán (Nariño) & Patia (Cauca) \\
\hline Cartagena del Chairá (Caquetá) & Tangua (Nariño) & Puerto Tejada (Cauca) \\
\hline Puerto Rico (Caquetá) & Abrego (Norte de Santander) & Santander De Quilichao (Cauca) \\
\hline San Vicente del Caguán (Caquetá) & Convención (Norte de Santander) & Aguachica (Cesar) \\
\hline Buenos Aires (Cauca) & El Tambo (Cauca) & Curumani (Cesar) \\
\hline Caloto (Cauca) & Jambalo (Cauca) & Corinto (Cauca) \\
\hline \multicolumn{3}{|c|}{ CONGLOMERADO TRES (3) } \\
\hline Municipio & Municipio & Municipio \\
\hline Medellin (Antioquia) & Montería (Córdoba) & Armenia (Quindio) \\
\hline Bello (Antioquia) & Soacha (Cundinamarca) & Pereira (Risaralda) \\
\hline Caucasia (Antioquia) & Quibdó (Choco) & Bucaramanga (Santander) \\
\hline Itagüi (Antioquia) & Neiva (Huila) & Barrancabermeja (Santander) \\
\hline Rionegro (Antioquia) & Riohacha (La Guajira) & Sincelejo (Sucre) \\
\hline Barranquilla (Atlántico) & Maicao (La Guajira) & Ibagué (Tolima) \\
\hline Soledad (Atlántico) & Santa Marta (Magdalena) & Cali (Valle) \\
\hline Bogotá, D.C. (Cund.) & Villavicencio (Meta) & Buenaventura (Valle) \\
\hline Cartagena (Bolivar) & Pasto (Nariño) & Jamundi (Valle) \\
\hline Manizales (Caldas) & Ipiales (Nariño) & Palmira (Valle) \\
\hline Florencia (Caquetá) & San Andrés de Tumaco (Nariño) & Tuluá (Valle) \\
\hline Popayán (Cauca) & Cúcuta (Norte de Santander) & Arauca (Arauca) \\
\hline Valledupar (Cesar) & Villa Del Rosario (Norte de Santander) & San José del Guaviare (Guaviare) \\
\hline
\end{tabular}

Fuente: elaboración propia.

\section{Notas}

* Artículo de investigación.

\section{Licencia Creative Commons CC BY 4.0}

Para citar este artículo: Rangel, H. S., Barrera, G., \& Gómez, O. M. (2021). Clasificación del riesgo de lavado de activos y financiación del terrorismo en Colombia en 2019. Cuadernos de Contabilidad, 22. https://doi .org/10.11144/Javeriana.cc22.crla 